Title of the article:

\title{
Surgical Experience with Chronic Constrictive Pericarditis
}

\section{Authors:}

Tsuneo Ariyoshi, MD, Kouji Hasizume, MD, Shinichiro Taniguchi, MD, Takashi Miura, MD, Kazuyoshi Tanigawa, MD, Seiji Matsukuma, MD, Tomohiro Odate, MD, Shun Nakaji, Mizuki Sumi, Kiyoyuki Eishi, MD.

Corresponding Author: Dr Tsuneo Ariyoshi,

Department of Cardiovascular Surgery, Nagasaki University School of Medicine, 1-7-1 Sakamoto, Nagasaki 852-8501, Japan.

Tel: 81958197307 Fax: 81958197311

Email: tsuneoariyoshi@infoseek.jp

Manuscript Types: Original Article

Word Count: 201 words in the abstract and 2195 words in the text.

Key word: Constrictive pericarditis, therapy 


\section{Abstract}

\section{Objective}

Constrictive pericarditis is a disease characterized by marked thickening of the pericardium which causes restriction of diastolic cardiac function. The purpose of this report is to review the outcome of pericardiectomy for constrictive pericarditis and to discuss its problems.

\section{Methods}

Sixteen consecutive patients who underwent pericardiectomy for constrictive pericarditis in our institution between March 2000 and June 2011 were reviewed. All patients underwent decortication including at least the anterior surface from the right atrium to the left phrenic nerve and the diaphragmatic surface of the heart. The epicardium was concomitantly resectioned because it was usually sclerotic and constrictive.

\section{Results}

Postoperatively, mean central venous pressure decreased to $8.8 \pm 3.1 \mathrm{mmHg}$ and was significantly lower than the preoperative value $15.3 \pm 3.7 \mathrm{mmHg}(\mathrm{p}<0.001)$.

The mean postoperative duration of hospitalization was $25.6 \pm 13.5$ days. There were 2 in-hospital deaths and early mortality rate was $12.5 \%$; however, excluding hospital 
T. Ariyoshi - 3

deaths, there were no significant postoperative complications and all survivors recovered and maintained good cardiac function during the mean follow-up period of $3.7 \pm 2.8$ years.

\section{Conclusion}

For an acceptable outcome, surgical intervention should be performed at an appropriate time and the pericardiectomy should have proper scope and depth to prevent irreversible changes in the heart, liver, and other organs. 


\section{Introduction}

Constrictive pericarditis is caused by inflammatory or non-inflammatory processes and is characterized by fibrotic and often calcific thickening of the pericardium. These changes of the pericardium cause restrictions in diastolic cardiac function leading to heart failure without pulmonary congestion.

Pericardiectomy for constrictive pericarditis was first successfully performed and described by Rehn et al. [1] and has become the accepted definitive method of treatment for improving cardiac hemodynamics [2-3].

In surgical treatment of constrictive pericarditis, decortication with an adequate scope and depth is necessary to restore normal cardiac function. However, there are some patients who show little improvement in diastolic cardiac function even with proper pericardiectomy.

The purpose of this report is to present our results of pericardiectomy for chronic constrictive pericarditis and to discuss several problems in this series of patients.

\section{Patients and methods}

From March 2000 to June 2011, consecutive 16 patients who underwent pericardiectomy for constrictive pericarditis in our institution were selected for analysis. 
The diagnosis of constrictive pericarditis was made on the basis of clinical and diagnostic criteria from radiological, electrocardiographic, echocardiographic, and right heart catheterization findings. Pericardiectomy was planned in a patient who had symptoms or signs related to certainly diagnosed constrictive pericarditis. Patients with newly diagnosed constrictive pericarditis had conservative management for several months before surgery, because constriction of pericardium may be transient in some cases.

Surgical and pathological findings were reviewed to confirm the preoperative diagnosis. Patients undergoing simultaneous pericardiectomy with surgical procedures for congenital or acquired heart disease and patients with pericardial effusion were excluded.

\section{Surgical procedure}

All patients underwent pericardiectomy through a midline sternotomy under cardiopulmonary bypass (CPB) assistance without cardiac arrest. Decortication of the anterior surface of the heart involving at least the area from the right atrium to the left phrenic nerve and the diaphragmatic surface was performed, except where calcification continued into the myocardium. Pericardiectomy was done as completely as possible; especially, great care was taken not to injure the phrenic nerves and the cardiac surface. 
We usually resect epicardium when it is constrictive (Figure1).

\section{Follow-up}

Postoperatively, digoxin or diuretics weaned at varying time intervals, however, when worsening of symptoms or signs from congestion were observed, these drugs were re-administered or increased. Follow-up was possible in all patients. All patients were contacted 2 weeks after discharge from the hospital, and their clinical conditions were documented. Patients returned to the clinic for follow-up visits yearly for a mean follow-up of $3.7 \pm 2.8$ years, and those who did not return to the clinic were contacted by phone for follow-up to document their conditions. All data are presented as mean \pm standard deviation (SD). Continuous scale data were analyzed using the Wilcoxon Signed Rank Test, and the Chi square test was used for categorical data. A p valự 0.05 was considered statistically significant.

\section{Results}

\section{Patient profiles}

Of these 16 patients, 14 (87.5\%) were male, and mean age was $62.9 \pm 13.6$ years with a range of 26 to 78 years. Seven patients (43.8\%) had dyspnea at rest. According to the New York Heart Association (NYHA) classification, 3 (18.8\%) patients had class IV 
heart failure, and 7 (43.8\%) patients had class III. Twelve patients (75.0\%) had peripheral edema, and computer tomography (CT) showed hepatomegaly in 3 patients (18.8\%), ascites in 8 (50.0\%) patients, and pleural effusion in 12 (75.0\%) patients. Preoperatively, 4 patients were on intravenous inotropic support, 3 patients on digitalis, and 15 patients on diuretics. Hypoalbuminemia (serum albumin $<3.5 \mathrm{mg} / \mathrm{dL}$ ) was detected in 4 patients and hyperbilirubinemia (serum bilirubin $>2.0 \mathrm{mg} / \mathrm{dL}$ ) in 6 patients (Table.1).

By the Child-Pugh score of liver failure, 6 patients were classified as class A, 10 as class B, and 1 as class C. On CT imaging, all patients had pericardial thickness greater than $5 \mathrm{~mm}$ in diameter. Calcific constrictive pericarditis was diagnosed in 5 (31.3\%) patients and some calcification in the myocardium was detected in 2 (12.5\%) patients on CT imaging. On electrocardiogram (ECG), low voltage was found in 8 patients.

All patients underwent right heart catheterization preoperatively, mean pulmonary artery pressure( PAP) was $23.3 \pm 4.4 \mathrm{mmHg}$, pulmonary capillary wedge pressure(PCWP) was $18.0 \pm 3.9 \mathrm{mmHg}$, left ventricular end-diastolic pressure(LVEDP) was $19.8 \pm$ 6.2mmHg, central venous pressure (CVP) was $15.3 \pm 3.7 \mathrm{mmHg}$ and right ventricular end-diastolic pressure (RVEDP) was $15.4 \pm 5.0 \mathrm{mmHg}$. All patients underwent echocardiography; the mean ejection fraction was $60.8 \pm 11.9 \%$. Three patients were found to have more than a mild tricuspid regurgitation (TR) (Table 2). 
Etiology of constrictive pericarditis

The cause of pericardial constriction could be identified in 11 patients.

One patient had tuberculous pericarditis, 2 patients had rheumatic pericarditis. 6 patients had a history of cardiac surgery (ranged 2 to 29 years), and 1 patient had undergone radiotherapy for mediastinal cancer. There was 1 constrictive pericarditis caused by Dressler`s post-myocardial infarction syndrome.

A specific etiologic factor could not be identified in the remaining 5 patients (Table2).

Postoperative results

Mean bleeding during operation was $2312 \pm 1311 \mathrm{ml}$ and mean transfusion of mannitol-adenine-phosphate (MAP) red cell, fresh frozen plasma (FFP) and platelet concentration (PC) was $6.5 \pm 5.3$ units, $4.8 \pm 4.3$ units and $6.6 \pm 9.8$ units, respectively. Postoperatively, 5 patients underwent right side heart catheterization, but CVP was measured in all patients. Mean CVP was $8.8 \pm 3.1 \mathrm{mmHg}$ and significantly lower than preoperative CVP $(\mathrm{p}<0.001)$; however, in 4 patients CVP was higher than $10 \mathrm{mmHg}$ even after decortication.

The duration of postoperative hospitalization was $25.6 \pm 13.5$ days, and at 2 weeks 
after discharge from the hospital, the condition of 6 patients was consistent with class I and 8 patients with class II according to NYHA classification (Table 3).

There were 2 in-hospital deaths and early mortality rate was $12.5 \%$. Of these 2 hospital deaths, Case 5 (Table.1) was a 59- year- old male who had a history of mitral valve replacement 20 years before the surgery and died 23 days after the surgery of multiple organ failure (MOF) caused by liver dysfunction which existed preoperatively and worsened postoperatively. His postoperative NYHA classification was class III.

The other death, Case 12 (Table.1) was a 61-year- old female who had a history of heart failure caused by rheumatic disease leading to decortication in 1980 . The extent of the previous pericardiectomy was very limited, only involving the anterior surface of the pericardium. She underwent pericardiectomy of the remaining thickened pericardium with concomitant resection of the epicardium from the anterior to the posterior surface but died 86 days after the operation due to MOF caused by persisting congestive heart failure. The preoperative CT image of this patients showed severe calcification in the lateral myocardial wall (Figure 2), and thus, complete pericardiectomy was impossible. Postoperative NYHA classification was IV and there was no improvement observed in CVP (CVP=17 mmHg) by right side heart catheterization. In the other patients radical pericardiectomy could be performed. 
Postoperative complications, excluding operative death, were limited to a re-intubation because of convulsion without major cerebrovascular accident, and a newly observed atrial fibrillation that was treated with drug therapy.

There were 4 late deaths, and 10 patients were alive as of the date of the last follow-up. The causes of late death were pneumonia in 1 patient (after 38.6 months), sepsis in 1(after 2.2 months) and cancer in 2 (after 37.6 and 18.6 months). Overall 1, 3 and 5 years survival rates were $92.9 \%, 84.4 \%$ and $65.7 \%$, respectively (Fig3).

At the most recent evaluation, 6 patients' conditions were consistent with NYHA class I and 4 patients with class II. There was no evidence of right sided heart failure in any of the patients.

\section{Discussion}

The relative prevalence of the causes of constrictive pericarditis have changed during the past few decades, in the past tuberculosis used to be the most frequent cause of constrictive pericarditis before the widespread use of antituberculosis chemotherapies. Pericarditis induced by purulent infection [5, 6] or collagen disease, especially rheumatoid arthritis [7] can be the cause of this disease. Constrictive pericarditis can also develop after cardiac surgery, and mantle chest radiotherapy [8, 9]. In this series of 
patients, constrictive pericarditis, which developed after open heart surgery, was the most frequently diagnosed cause and tuberculosis was found only in 1 patient.

It is expected that the number of constrictive pericarditis cases following cardiac surgery will increase, because cardiac surgery has become more frequent during the last decade. In patients with constrictive pericarditis following cardiac surgery, diffuse calcification of pericardium is seen much less commonly than patients with tuberculosis.

However, after an open heart surgery, not only constrictive pericarditis but also myocardial restrictive disease can develop.

Radiotherapy is another increasing cause of this disease and reported one of poor prognostic factors after surgery [8, 9]. Because pericardectomy has a significant operative mortality[10], the recommendation of surgical intervention should be made carefully in such a patient. In our series, however, only one patient had a history of mediastinal radiation therapy.

Radical pericardiectomy involves wide resection of the anterior surface of the heart between the phrenic nerves and the diaphragmatic surface. Other smaller excisions with more limited scope are considered to be partial. The extent of pericardiectomy needed for constrictive pericarditis has been discussed [3-4, 10-11]. The point at discussion is the 
necessity of decortication of the right atrium and the vena cava.

In our institution, decortications are performed from the right atrium to the left phrenic nerve and the diaphragmatic surface.

When severe pericardial constriction exists, decortication of the right atrium may be necessary. Sufficient decrease in right atrial pressure may not occur without decortication of the right atrium, and this may cause congestion in the extremities and lead to hepatic and other organ dysfunction. Thus, we believe our strategy of the extent of decortication is appropriate not only to recover the diastolic cardiac function but also to improve right atrial (RA) function in the systolic phase.

The second concern of the surgical procedure is the depth of decortication. Because epicardial sclerosis could be a cause of a delayed response or persistent hemodynamic abnormalities, even when adequate pericardiectomy is performed [12]. Resection of the epicardium is necessary in addition to pericardiectomy for chronic constrictive pericarditis. However, it may be difficult to excise sclerotic epicardium adequately without any injury to the cardiac surface because sclerotic epicardium is constrictive, but it is usually thin and nearly transparent like a sheet of cellophane.

The Waffle procedure originally reported by Heimbecker and colleagues [13] is an 
effective procedure for constrictive and sclerotic epicardium, but the outcome of this method in the long term is not yet clear. We routinely aim to perform excision of the epicardium as completely as possible. With our strategy, all survivors in this series had a significant decrease in their CVP and their conditions were consistent with class I or II according to NYHA classification, two weeks after discharge from the hospital and at the most recent follow-up.

We perform surgical intervention under $\mathrm{CPB}$ assistance via median sternotomy to achieve pericardiectomy of adequate extent and depth.

Although left thoracotomy is less invasive than median sternotomy, we prefer the median approach. A median approach allows easier resection of the pericardium overlying the thin-walled RA and good access to all areas that should be treated. We believe that use of CPB makes it possible to perform a safer operation, even when fatal bleeding due to atrial or ventricular injury occurs. In addition, a more adequate and easier epicardectomy can be performed, especially in patients who have a history of cardiac surgery, though the use of CPB could increase the risk of bleeding during surgery.

The last concern is the timing of the surgical intervention. To decide appropriate timing of surgical intervention, early and accurate diagnosis is necessary. In addition, exact 
evaluation of the degree to which the abnormal pericardium contributes to increased intracardiac pressure is also needed.

The diagnosis of constrictive pericarditis is often difficult because of its different clinical presentations; thus, the most important diagnostic point may be the clinical suspicion of constrictive pericarditis in a patient with right sided heart failure that is disproportionate to pulmonary or left sided heart disease.

Echocardiography is a non-invasive and very useful diagnostic tool. A septal bounce, respiratory septal shift in 2-dimensional echocardiogram [14], and inspiratory decreases in the initial E velocity on the mitral inflow velocity curve in Doppler interrogation [15], are useful for confirming the diagnosis. Also, an echocardiogram may provide information of other cardiac disorders.

CT and magnetic resonance imaging (MRI) enable direct visualization of the pericardium and are useful tool for diagnosis [16-19]. Each of these methods detects an increase in pericardial thickness of greater than $2 \mathrm{~mm}$ in diameter.

In this series, there were 2 in-hospital deaths; 1 patient died of MOF resulting from liver dysfunction which worsened postoperatively, and the other died of MOF resulting from persistent hemodynamic abnormalities.

Rienmüller et al [19] reported the use of CT and/or MRI in 80 patients with pericardial 
constriction confirmed by catheterization data. In their report, 7 patients who had myocardial atrophy and/or fibrosis underwent pericardiectomy and all died of myocardial failure. Tirilomis et al [20] documented the results of 71 consecutive patients who underwent pericardiectomy and recommended early pericardiectomy. They concluded that the outcome is related not only to the extent of surgery, but also to myocardial involvement, because of prolongation of pericardial constriction or inflammatory processes extending into the myocardium resulting in myocardial insufficiency. In fact, myocardial fibrosis often exists in constrictive pericarditis [21]. MRI can give us information about pericardial inflammation and pericardial- myocaldial adherence as well as myocardial fibrosis [17].

On the other hand, with regard to hepatic dysfunction, it was reported that reversibility of hepatic function after surgical intervention for constrictive pericarditis might be dependent on the duration of liver congestion [22].

Preoperatively, both patients who died during their hospitalization had high Child-Pugh scores (=9) and had a long history of constrictive pericarditis.

Their hepatic function could not be restored even after decortications, and one of them could not undergo radical pericardiectomy because of calcification in the myocardium.

Postoperatively, their CVP did not decrease to below $10 \mathrm{mmHg}$ and hemodynamic 
abnormalities persisted.

In contrast to these 2 patients, the others recovered their cardiac function. Thus, the 2 patients who died should have undergone earlier surgical repair to improve their outcomes.

We suggest that early pericardiectomy should be planned to improve results, however reversible constrictive pericarditis [23] should be excluded before the surgical intervention. For this purpose, patients with newly diagnosed constrictive pericarditis could be treated conservatively and further diagnostic examination using MRI may be useful[17-18].

In conclusion, although accurate diagnosis of constrictive pericarditis is often difficult because of its various clinical presentations, earlier operation based on exact diagnosis is needed to improve cardiac function.

When pericardiectomy of proper extent and depth with suitable timing is performed for patients with constrictive pericarditis, a favorable outcome can be expected.

Conflict of interest

None 


\section{References}

1 Rehn, L., and Sauerbruch, F.: Cited by White, P.D.: Chronic constrictive pericarditis( Pick`s disease) treated by pericardial resection. Lancet 1935; 2: 539 and 537.

2 Harrison EC, Crawford DW, Lau FY. Sequential left ventricular function studies before and after pericardiectomy for constrictive pericarditis. Delayed Resolution of residual restriction. Am J Cardiol 1970; 26(3): 319- 23.

3 Kloster FE, Crislip RL, Bristow JD, Herr RH, Ritzmann LW, Griswold HE. Hemodynamic studies following pericardiectomy for constrictive pericarditis. Circulation 1965; 32(3):415-24.

4 Lazarides DP, Avgoustakis DG, Lekos D, Michaelides GB: Evaluation of radical pericardiectomy for constrictive pericarditis: A clinical, hemodynamic and electrocardiographic study of twenty cases. J Thorac Cardiovasc Surg 1966; 51(6):821-33.

5 Das PB, Gupta RP, Sukumar IP, Cherian G, John S. Pericardiectomy. Indications and results. J Thorac Cardiovasc Surg 1973; 66(1): 58 -70.

6 Majid AA, Omar A. Diagnosis and management of purulent pericarditis. Experience with pericardiectomy. J Thorac Cardiovasc Surg 1991; 102(3): 413-7.

7 Burney DP, Martin CE, Thomas CS, Fisher RD, Bender HW. Rheumatoid pericarditis. 
Clinical significance and operative management. J Thorac Cardiovasc Surg 1979; 77(4):511-5.

8. Ling LH, Oh JK, Schaff HV, Danielson GK, Mahoney DW, Seward JB, et al. Constrictive pericarditis in the modern era: evolving clinical spectrum and impact on outcome after pericardiectomy. Circulation 1999; 100:1380-6.

9 Bertog SC, Thambidorai SK, Parakh K, Schoenhagen P, Ozduran V, Houghtaling PL, et al. Constrictive pericarditis: etiology and cause-specific survival after pericardiectomy. J Am Coll Cardiol 2004;43:1445-52.

10 Chowdhury UK, Subramaniam GK, Kumar AS, Arian B, Singh R, Talwar S, et al. et al. Pericardiectomy for constrictive pericarditisa clinical, echocardiographic, and hemodynamic evaluation of two surgical techniques. Ann Thorac Surg 2006;81:522-530. 11 Viola AR. The influence of pericardiectomy on the hemodynamics of chronic constrictive pericarditis. Circulation 1973;48(5) :1038-42.

12 Walsh TJ, Baughman KL, Gardner TJ, Bulkley BH. Constrictive epicarditis as a cause of delayed or absent response to pericardiectomy: a clinicopathological study. J Thorac Cardiovasc Surg 1982; 83(1) :126-32.

13Heimbecker RO, Smith D, Shimizu S, Kestle J. Surgical technique for the management of constrictive epicarditis complicating constrictive pericarditis (the waffle procedure). 
Ann Thorac Surg 1983; 36(5):605-6.

14 Himelman RB, Lee E, Schiller NB. Septal bounce, vena cava plethora, and pericardial adhesion: informative two-dimensional echocardiographic signs in the diagnosis of pericardial constriction. J Am Soc Echocardiogr. 1988; 1(5):333-40.

15 Oh JK, Hatle LK, Seward JB, Danielson GK, Schaff HV, Reeder GS, et al. Diagnostic role of Doppler echocardiography in constrictive pericarditis. J Am Coll Cardiol. 1994 Jan;23(1):154-62.

16 Breen JF. Imaging of the pericardium. J Thorac Imaging 2001; 16(1):47-54.

17 Verhaert D, Gabriel RS, Johnston D, Lytle BW, Desai MY, Klein AL. The role of multimodality imaging in the management of pericardial disease. Circ Cardiovasc Imaging 2010;3:333-43.

18 Feng D, Glockner J, Kim K, Martinez M, Syed IS, Araoz P, et al. Cardiac magnetic resonance imaging pericardial late gadolinium enhancement and elevated inflammatory markers can predict the reversibility of constrictive pericarditis after antiinflammatory medical therapy: a pilot study. Circulation. 2011 Oct 25;124(17):1830-7.

19 Rienmüller R, Gürgan M, Erdmann E, Kemkes BM, Kreutzer E, Weinhold C. CT and MR evaluation of pericardial constriction: A new diagnostic and therapeutic concept. J 
Thorac Imaging 1993; 8: 108-121.

20Tirilomis T, Unverdorben S, von der Emde J. Pericardectomy for chronic constrictive pericarditis: risks and outcome. Eur J Cardiothorac Surg 1994; 8(9): 487-92.

21 Levine HD. Myocardial fibrosis in constrictive pericarditis. Electrocardiographic and pathologic observations. Circulation 1973; 48(6) :1268- 81.

22 Tagami H, Utoh J, Kunitomo R, Hagiwara S, Uemura S, Muranaka T, et al. Hepatic function after surgical repair of constrictive pericarditis. Kyobu geka. The Japanese journal of thoracic surgery 2000; 53(7): 567-70.

23 Haley JH, Tajik AJ, Danielson GK, Schaff HV, Mulvagh SL, Oh JK. Transient constrictive pericarditis: causes and natural history. J Am Coll Cardiol 2004;43:271-5. 
T. Ariyoshi - 21

\section{Figure legends}

\section{Figure 1}

The resection of constrictive epicardium. Arrows show the thickened pericardium.

\section{Figure 2}

On CT imaging, severe calcification was seen to extend into the myocardium.

\section{Figure3}

Actuarial survival rate after surgery 
Figure 1

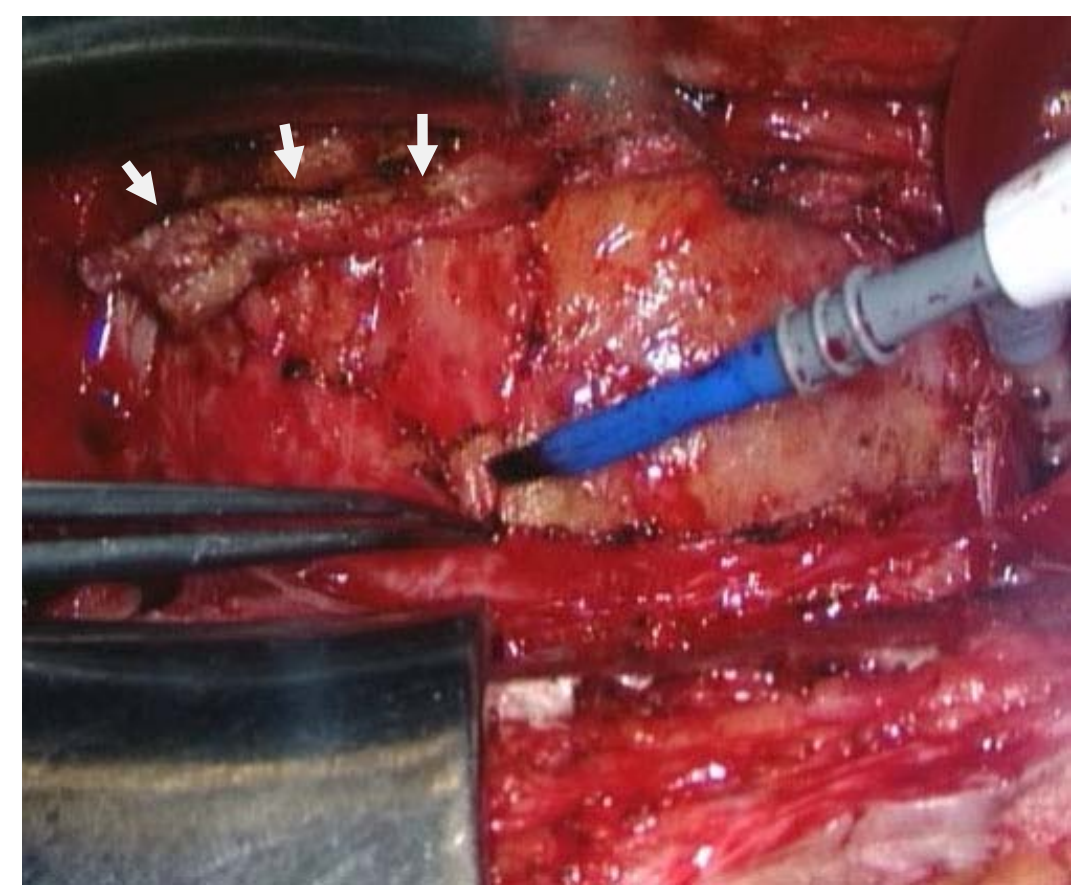


Figure 2

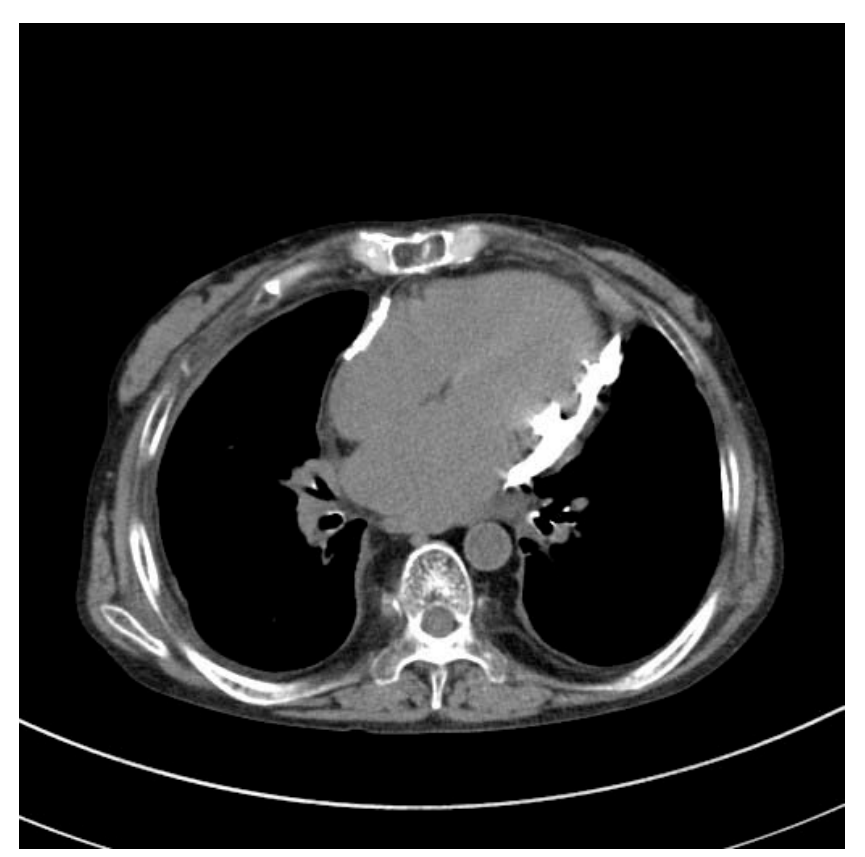


Figure 3

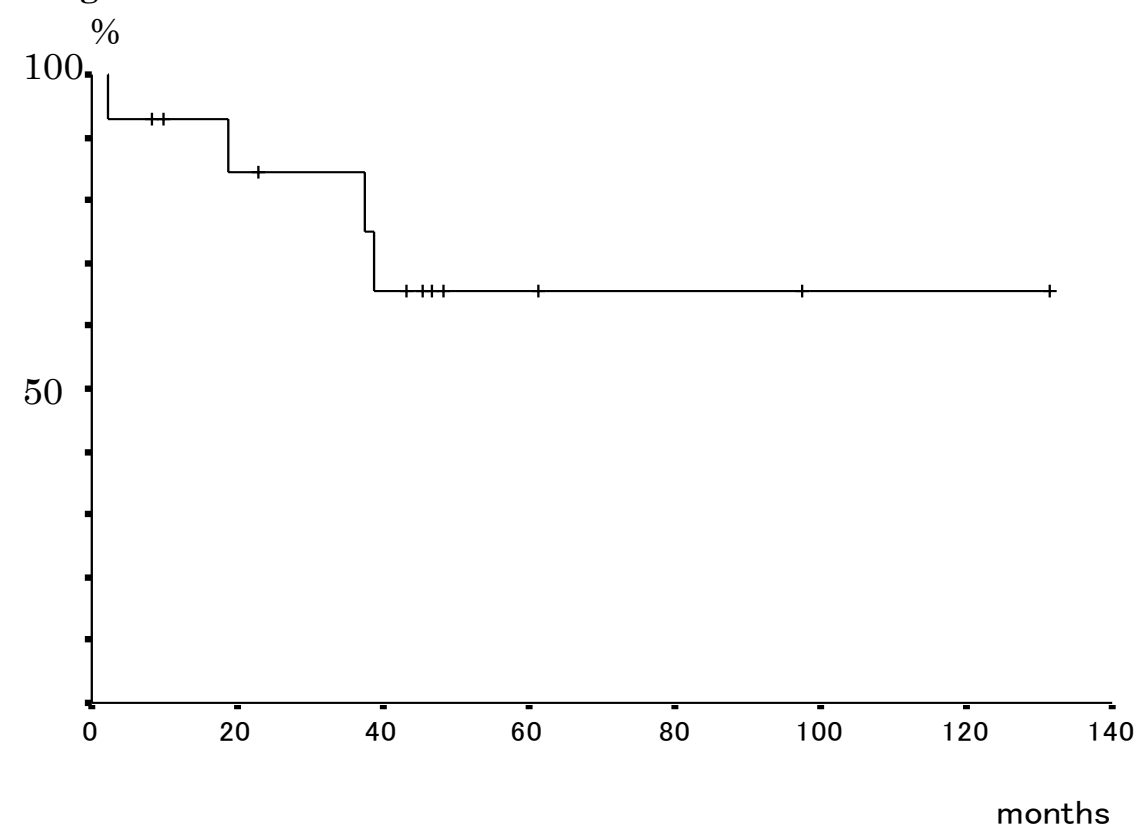


Table 1 Preoperative characteristics of patients

\begin{tabular}{|c|c|c|c|c|c|c|c|c|c|c|c|c|c|c|c|}
\hline \multirow{2}{*}{ No } & \multirow{2}{*}{ age } & \multirow{2}{*}{ sex } & \multirow{2}{*}{ Cause } & \multirow{2}{*}{ NYHA } & \multirow{2}{*}{$\begin{array}{c}\text { Dyspnea } \\
\text { at rest }\end{array}$} & \multirow{2}{*}{ Hepatomegaly } & \multirow{2}{*}{ ascites } & \multirow{2}{*}{$\begin{array}{c}\text { Peripheral } \\
\text { edema }\end{array}$} & \multirow{2}{*}{$\begin{array}{l}\text { Pleural } \\
\text { effusion }\end{array}$} & \multirow{2}{*}{ T-bil } & \multirow{2}{*}{ Alb } & \multirow{2}{*}{$\begin{array}{c}\text { Inotoropic } \\
\text { support }\end{array}$} & \multicolumn{3}{|c|}{ Mediation } \\
\hline & & & & & & & & & & & & & diuretics & digitalis & steroid \\
\hline 1 & 72 & M & Cardiac surgery: AVR(1994) & IV & 1 & 0 & 0 & 1 & 1 & 1.1 & 3.8 & 0 & 1 & 0 & 0 \\
\hline 2 & 40 & M & Non-specific & II & 0 & 0 & 1 & 0 & 0 & 0.7 & 4.8 & 0 & 0 & 0 & 0 \\
\hline 3 & 54 & M & Non-specific & II & 0 & 0 & 0 & 1 & 0 & 2 & 3.8 & 0 & 1 & 0 & 0 \\
\hline 4 & 72 & $\mathrm{~F}$ & Radiotherapy & III & 0 & 0 & 0 & 1 & 1 & 2.1 & 3.7 & 0 & 1 & 1 & 0 \\
\hline 5 & 59 & M & Cardiac surgery: MVR (1984) & IV & 0 & 1 & 0 & 0 & 1 & 4 & 3.9 & 1 & 1 & 1 & 0 \\
\hline 6 & 26 & M & Tuberculosis & II & 0 & 0 & 0 & 1 & 1 & 3.2 & 4.5 & 0 & 1 & 0 & 1 \\
\hline 7 & 67 & M & Cardiac surgery: MVR+TAP(2005) & III & 1 & 1 & 1 & 1 & 1 & 2.9 & 3.6 & 1 & 1 & 0 & 0 \\
\hline 8 & 75 & M & Non-specific & II & 0 & 0 & 0 & 1 & 1 & 1.7 & 3 & 0 & 1 & 0 & 0 \\
\hline 9 & 58 & M & Non-specific & III & 1 & 0 & 0 & 0 & 1 & 0.9 & 3.8 & 0 & 1 & 0 & 0 \\
\hline 10 & 76 & M & Cardiac surgery: CABG(2003) & II & 1 & 0 & 0 & 1 & 1 & 0.8 & 4.1 & 0 & 1 & 0 & 0 \\
\hline 11 & 64 & M & Cardiac surgery: CABG(2005) & II & 0 & 0 & 0 & 1 & 0 & 1.3 & 4.4 & 0 & 1 & 0 & 0 \\
\hline 12 & 61 & $\mathrm{~F}$ & Rheumatic: decortication(1980) & IV & 1 & 1 & 1 & 1 & 1 & 0.6 & 2.2 & 1 & 1 & 1 & 1 \\
\hline 13 & 56 & M & Non-specific & I & 0 & 0 & 0 & 0 & 0 & 1.4 & 4.4 & 0 & 1 & 0 & 0 \\
\hline 14 & 74 & M & Rheumatic disease & III & 1 & 0 & 0 & 1 & 1 & 3.1 & 2.4 & 0 & 1 & 0 & 1 \\
\hline 15 & 78 & M & Cardiac surgery: MVP (2003) & III & 1 & 0 & 0 & 1 & 1 & 1.1 & 3.4 & 0 & 1 & 0 & 0 \\
\hline 16 & 68 & $\mathrm{M}$ & Myocardial infarction & III & 0 & 0 & 1 & 1 & 1 & 1.4 & 3.8 & 1 & 1 & 0 & 0 \\
\hline
\end{tabular}

NYHA, New York Heart Association; T-Bil, total bilirubin; Alb, albumin; AVR, aortic valve replacement; MVR, mitral valve replacement; CABG, coronary artery bypass grafting; MVP, mitral valve plasty; TAP, tricuspid annuloplasty. 
Table 2. Preoperative status of patients

patient profiles

Total number of patients

Age

$62.9 \pm 13.6(26-78)$

Sex (Male/Female)

$14 / 2$

Child-Pugh classification

Grade A

Grade B

Grade C

Child-Pugh score

$7.3 \pm 1.4$

Calcific constrictive pericarditis

Myocardial calcification

Etiology of chronic constrictive pericarditis

Following Cardiac surgery

Unknown

Rheumatic disease

Tuberculosis

Radiotherapy

Myocardial infarction(Dressler`s syndrome) 
NYHA classification

$\begin{array}{lll} & \text { II } & 6 \\ & \text { III } & 7 \\ \text { Atrial fibrillation } & \text { IV } & 3 \\ & & 4\end{array}$

$\mathrm{EF}(\%)$

$60.8 \pm 11.9$

TR

mild to moderate $\quad 2$

moderate

mPAP (mmHg) $\quad 23.3 \pm 4.4$

PCWP (mmHg) $\quad \underline{18.0 \pm 3.9}$

$\begin{array}{lr}\text { LVEDP (mmHg) } & 19.8 \pm 6.2\end{array}$

$\begin{array}{lr}\text { CVP (mmHg) } & 15.3 \pm 3.7\end{array}$

RVEDP (mmHg)

$15.4 \pm 5.0$

T-Bil, total bilirubin; Alb, albumin; NYHA, New York Heart Association; EF, ejection fraction; TR, tricuspid regurgitation; mPAP, mean pulmonary artery pressure; PCWP, pulmonary capillary wedge pressure; LVEDP, left ventricular end-diastolic pressure; CVP, central venous pressure; RVEDP,

right ventricular end-diastolic pressure 
Table 3. Pre-and postoperative cardiac status

\begin{tabular}{|c|c|c|c|c|}
\hline Variables & & preoperative & postoperative & $\mathrm{p}$ value \\
\hline \multirow[t]{4}{*}{ NYHA classification } & & & & 0.008 \\
\hline & I & 0 & 6 & \\
\hline & II & 6 & 8 & \\
\hline & III & 7 & 1 & \\
\hline
\end{tabular}

NYHA, New York Heart Association; CVP, central venous pressure 